opening made in the duct, and the junction reinforced by drainage wicks of gauze brought ont through the abdominal incision, the whole arrangement being surrounded and isolated by omentum carefully fixed by sutures. The gauze acts as a drain for any leaking pancreatic secretion, and further sets up firm omental adhesions before its withdrawal at the end of two or three days. Neglect to isolate the drainage aperture in the way indicated may lead to serious or fatal results, for the secretion is not only in itself capable of necrosing effects on the peritoneum, but is in addition germ infected in many cases of pancreatitis. Drainage of the pancreas by incisions into the gland itself, while necessary in desperate cases of acute pancreatitis, is of doubtful expediency in chronio cases. If employed, it should be curried out only by means of the freest combination of large rubber tubes and ample gauze packing carefully surrounded by omentum, and, if at all possible, by the gastro-colic route, which best lends itself to such an arrangement.

\section{THE CAUSATION AND TREATMENT OF RICKETS.}

BY

ERIC PRITCHARD, M.A., M.D.Oxon., M.R.C.P.Lond.,

PHYSICIAN TO THE QUEEN'B HOSPITAL FOR CHILDREN AND TO THE CTTY OF LONDON HOSPITAL FOR DISEASEB OF THE CHEST.

IT is to my mind open to question whether the time has not now arrived for a reconsideration of the whole subject of rickets-its definition, its etiology, and its treatment.

The following may be taken as a typical definition of the disease:

Rickets is a chronic general disorder of nutrition occurring during infancy from faulty diet and hygienic defloiencies. It involves the whole body, but its most obvious effects are recorded in the skeletal system.

This definition, and the majority of those with which I am acquainted, appear to me to confuse cause and effect. If the term is not confined to the bony changes, but covers also all the various forms of malnutrition which ultimately terminate in rickets, the name means little else than a generic one for ill health in infants and young children. Some authorities classify such symptoms as sweating, muscular weakness, laxity of ligaments, delayed teething, spasmophilic manifestations, and tendencies to catarrhs as rachitic. Francis Glisson included in his famous monograph on rickets every symptom and childish ailment which was known in his day.

The view which I propose to develop in this lecture is that practically all varieties of malnutrition occurring during infancy and early childhood tend to terminate in rickets, provided they are sufficiently severe or long en ugh continued. They should not, however, be regarded as evidence of rickets unless they are actually accompanied by the typioal changes in bone which are characteristic of the disease.

Each variety of malnutrition has its own particular etiology and requires special lines of treatment, and cannot be dealt with rationally on the cut-and-dried lines that are usually advocated for rickets itself.

Shortly stated, the theory which I wish to propound is that the essential and central feature of rickets is the want of calcification or mineralization of developing bone, and that this in its turn is due to the existence of require ments for calcium, which for the time being are more urgent than those of developing bone. These urgent requirements are the necessity for neutralizing acid bodies in the blood; in other words, to neutralize or compensate an existing acidosis. My argument is that all chronic conditions of malnutrition, of whatever kind or from whatsoever cause arising, finally terminate in an acidosis, and that all claims on alkaline bases arising in connexion with the neutralization of this acidosis must be satisfied before those of developing bone are attended to.

It is not difficult to understand why all forms of mal. nutrition tend to end in a condition of acidosis, for in all cases, though in various ways, metabolic or oxidation processes are interfered with and curtailed. Any interference with the due oxidation of food elements to their normal * A lecture delivered before the Fellowship of Medicine at the Royal
Society of Modicine, October lst, 1919. end-products, carbonic acid, urea and water, will conduce to the formation of semi-oxidized bodies of large molecular size and acid in reaction, which must be neutralized as quickly as they are formed to prevent a dangerous rise in the hydrogen ion concentration of the blood. It is in the satisfaction of these claims for alkaline bases that the injury is done to growing bone. One of the direct causes of an acidosis is a disturbance of the relationship between the caloric value of the food consumed and the actual output of energy, either in the form of heat or of work. If more food is consumed than is required for the physiological purposes of the body there will be an attempt to adjust this want of balance by excreting part of the food in an unoxidized, or semi-oxidized, condition. Any condition, therefore, which tends to diminish the efficiency of the human body as a working machine will tend to reduce the physiological demands for food, and so renders a pre-existing correct diet thereby excessive, or relatively excessive.

Most of the recognized etiological factors of rickets are factors which interfere in some way with the metabolic or oxidizing processes of the body-in other words, interfere with the proper utilization of food. Any condition, therefore, which interferes with the supply of oxygen will tend in the long run to be a factor in the production of rickets. So also will be any condition which interferes with the output of work-as, for instance, the poisoning or degeneration of nerve cells. Anything also which unduly interferes with the dissipation of heat -as, for instance, warm rooms or excessive clothing. The withholding of surface stimulation, sach as is afforded by sunshine and currents of fresh air, will also have the same effect. Most of the etiological factors of rickets are included in this category of causative factors of an acidosis; and, in the light of these explanations, I pro. pose now to review some of the theories on the pathogenesis of rickets which have been formulated from time to time, and which for the most part have been unwar. rantably rejected as unsatisfactory, because, although possibly applying to certain cases, fail to do so to all. I shall hope to show that while there are different theories required to explain the various forms of malnutrition which are fore-stages in the common terminal resultnamely, the production of an acidosis-there is only one explanation of the want of calcification of bone, and that is the urgent requirement of the body to have the acidosis neutralized at the expense of the alkaline reserves of the blood, reserves from which growing bone would otherwise draw its mineral material.

The first theory to be considered is that of a deficiency of lime in the food. It is clear, apart from any experimental proof, that without lime bones cannot be formed, but since there is an adequate supply of calcium in breast milk, and an excess of this base in cow's milk, it is obvious that want of this element cannot per se be the explanation of rickets in the majority of cases. None the less, a deficiency of lime is quite probably the true explanation of a small minority of cases in which certain patent foods are exclusively employed.

Defective absorption of lime is a more reasonable ex. planation, and colour is lent to this theory because in rickets an increased output of lime in the faeces can practically always be proved.

It is easy to understand that any available base such as calcium existing in the intestinal contents will be readily seized to neutralize any acid bodies formed as the result of putrefactive or fermentative changes in the bowel. It has been denied that this is the true explanation of rickets, for the reason that the administration of sup. plementary supplies of calcium salts in rachitic con. ditions is followed by an equivalent output of calcium in the urine. This to my mind proves nothing, for if under such conditions excess of calcium is absorbed into the blood, it may well be utilized for the neutralization of acid bodies coexisting in the blood. It is interesting to note that soap stools are very common in rickets, and these soaps consist largely of fatty acids in combination with calcium, a fact which is very suggestive of the occurrence of an excess of fatty acids in the bowel in the conditions which predipose to rickets. But whether calcium is eliminated in excess or not in the bowel, there is abundant evidence to show that it is eliminated in excess either in the faeces or in the urine, or in both. 
Intestinal intoxication has been regarded as an important factor in rickets, and there can be little doubt that there are many reasons for accepting this as one of the causes. Toxaemias of this kind are responsible for serious poisoning of the nervous system, for nerve cell degeneration, and for functional impairment of the organic functions in general, and for those of the muscular system in particular. 'The fact that severe rickets can occur without evidence of intestinal intoxication does not militate against the view that it does so in a certain number of cases.

Defective action of the endocrine glands has been sug. gested by many observers as the causa causans of rickets. There is much evidence to support the view that disturb. ances of the thyroid, parathyroid, adrenal, or pituitary glands are occasionally concerned in the rachitic processes. It is impossible to believe that such disturbances should be entirely absent in the great variety of conditions of malnutrition which ultimately cause unequivocal symptoms of rickets; but it is extremely improbable that in so common a disease as rickets any one glandular disturbance can be regarded as the primary factor. Moreover, rickets is a short-lived disease, while disease or disturbance of the functions of any of these glands is liable to persist. That the thyroid apparatus is concerned in calcium metabolism cannot be doubted; moreover, hyperthyroidism is apparently a compensatory phenomenon in septic conditions which may in themselves be a cause of acidosis and rickets. The adrenals undergo degenerative processes in those conditions of malnutrition which follow on deprivation of the accessory food factors (MeCarrison).

Cassowitz believes that deleterious respiratory influences, or bad air (odour of the poor), play a direct part in the etiology of rickets, and bases this hypothesis on the rise in the incidence curve of rickets after the winter months. Foul air must necessarily depress vital functions, and so also must want of stimulation by currents of fresh cold air (Leonard Hill). Hence it would be contrary to my own theory if want of ventilation did not play an occasional part as a causative agent.

Dr. L. Findlay has conducted some experiments on animals to prove that want of muscular exercise plays an important part in bricging about the rickety condition. This entirely coincides with my own practical experiences in connexion with the management of infants. Exercise creates a demand for energy which is greater than all the other organic demands of the body taken in combination. If there is no ( $u$ ' put of kinetic energy, as in debilitated states, or from restraint owing to tight clothing, the physiological uses of food will be seriously restricted. There will be lessened incentive for oxidation processes, incomplete combustion, and a corresponding output of acids of large molecular size-in other words, with restriction of exercise an otherwise physiological dietary becomes at once relatively excessive.

Within recent years great importance has been attached to the part which the accessory food factors (vitamines) play in the determination of rickets. Three distinctive varieties of malnutrition have been recognized as due to starvation in respect of the three vitamines so far dis covered. These are, first, infantile scurvy from want of the water-soluble accessory factor " $C$ "; secondly, arrest of growth for want of the fat-soluble accessory factor "A"; and thirdly, a variety of malnutrition analogous to beriberi, and which is due to the want of the autineuritic vitamine, the water-soluble " $B$ " factor contained in the cortical part of cereal grains. This latter form of malnutrition only arises in infants in whom decorticated cerea foods are largely employed. According to my view any one of these forms of malnutrition will ultimately lead to an acidosis, and consequently to the rickety condition, but in each case the distinctive features of the special variety 0 " malnutrition will lend special character to the clinical picture. Hopes have recently been raised that want of the fat soluble vitamine contained in animal oils is the cause of rickets; if this hope were justified the distribution of a little cod-liver oil would free all civilized countries from the scourge of one of the commonest diseases. It is perhaps unnecessary to point out that if this hypothesis be true it is difficult to explain the reason for rickets amongst well-to-do children fed on an excess of cream.

Acid conditions of the blood or the excessive formation of acid bodies has frequently been claimed to be the cause of rickets. As I have already indicated, an acid condition of the blood is incompatible with life; and the most delicate and efficient mechanisms exist for the main tenance of a constant and uniform degree of hydrogen ion concentration. No free acids can exist in the blood; they are at once neutralized at the expense of the alkali reserves. The first of these theories was that of Wachsmuth, who believed that the trouble in rickets was due to an excessive production of carbonic acid, which he believed was capable of depriving bone of its mineral rights. 'This theory is unworthy of serious consideration in view of simple physical and physiological knowledge. The lactic acid theory is quite inadequate to explain all the phenomena connected with the production of rachitic changes in the bone. Lactic acid is certainly produced in excess under conditions of restricted oxidation (anoxaemia), but this acid only represents one among many acids of large molecular size which are inevitably produced under these conditions. I pointed out as early as 1901, in a paper read at the British Medical Association's annual meeting held at Cheltenliam, that the relative overfeeding of infants must necessarily lead to the production of a large number of acid bodies which would draw upon the alkaline reserves of the blood, and thins tend to produce a condition of acidosis. Since then Stóltzner has elaborated his "oxypathic theory" of rickets which is very much the same as my own, although he does not harmonize it satisfactorily with all the know etiological factors of rickets.

An argument commonly urged against the acidosis theory is that it is inconceivable that acid bodies could exist in the blood sufficiently strong to dissolve tlie fixed salts out of bone.' 'This is quite beside the question as far as rickets is concerned, for in this disease we are dealing with the withlolding of the mineral material from bone which is in process of formation, and not with its extraction from completely developed bone, as is the case in osteo. malacia, for instance. Further, it has been argued that if in rickets calcium is used up in neutralizing acid bodies, the amount of calcium in the blood should show a noticeable falling off in its calcium content, which has not, so far, been capable of demonstration. No doubt calcium would be among the last of the basic elements which constitute the alkali reserve in the blood to be us $d$ up for neutralization purposes; but in rickets there is an increase of the calciuin in the urine, proving that calciun is to some extent used up and withlield from its normal destinations, even though there is no diminution in the blood.

Quite recently it has been show wh that in tetany, which may be regarded as evidence of a severe degree of acidosis and a phenomenon of advanced ríckets, there is a demon. strable falling off in the calcium content of the blood, ${ }^{1}$ a piece of evidence which strong $\mid y$ supports my views on the etiology of rickets.

In any long continued acidosis it is logical to expect that among other elements iron itself will-be seized upon for neutralization purposes, in which event a corresponding amount of iron must be withlield from developing blood corpuscles. This may explain a coexisting anaemia, and the compensatory activities of the red marrow and other blood-forming centres. On this explanation we can understanding the beading of ribs, and the enlargement of the epiphysis of long bones.

Many investigations have been made to discover a bacterial origin of rickets. This seems to me a useless waste of time, for all infective and septic processes lead to a condition of acidosis, and there is no need to search for a specific bacillus of rickets when practically any variety is capable of producing the disease. Chronic infections, such as tuberculosis, syphilis, malaria, and those due to the Bacillus coli, almost invariably lead in infants to rachitic manifestations.

From the foregoing it is obvious that many etiological theories are required to explain the causation of the many varieties of malnutrition which are antecedent to the rachitic changes in the bones, but all of them lead to this goal through the common avenue of acidosis. From a practical point of view it is most desirable that we should recognize what are the common and what are the rare etiological factors which finally lead to the bony changes of rickets.

In different countries and under different conditions varying fact $n$ rs will be found to predominate. In this country, and especially among the urbeh population I imagine that catarrhal and other infections play a most important rôle. Impure air, want of sunshine, want of 
exercise, and want of surface stimulation - in other words, bad housing conditions-also play important parts, and conduce to that condition of relative overfeeding which I have insisted on as the essential element in leading to acidosis. Among the well-to do classes overfeeding and under-exercise are the dual causes.

The treatment of rickets naturally resolves itself into prophylactic and remedial measures. Prophylaxis is concerned with the avoidance of those malhygienic conditions which favour the development of the various forms of malnutrition which have already been described. Correct dieting is essential to avoid catarrhal conditions of the bowel, intestinal toxaemias, and insufficiency diseases, all of which by their respective routes lead to the common goal-acidosis. Food in excess, even though otherwise beyond criticism, is as dangerous as unsuitable food itself, for in the long run it necessitates a short-circuiting of the metabolic processes and the production of acids of large molecular size, such as uric, oxalic, butyric, lactic, propionic, and to some extent of such powerful acids as sulphuric and phosphoric, all of which must be neutralized at the expense of ammonium and other altali reserves of the blood including calcium. Any condition which interferes with oxidation will similarly restrict the completeness of the metabolic processes, and anong them must be included want of fresh air, mechanical obstruction of the respiratory passages by adenoids, embarrassments of the respiratory movements, as well as all other conditions which lead to anoxaemia. Want of stimulation of the skin surface by cool air, good ventilation, or baths, will similarly restrict the oxidation processes.

Want of exercise-as, for instance, by the limitation of muscular activities, such as those concerned in playing, kicking, rolling, and walking-will also predispose to the same results; in fact muscular exercise is the grand prophylactic against rickets. Unfortunately, in the majority of cases of malnutrition which terminate in rickets, active exercise is limited owing to nervous depression, nuclear degenerations, and muscular debility, but none the less every opportunity to ensure free exercise must be provided for all healthy infants. No matter how correctly fed, or how good the other hygienic conditions, infants who are swaddled up in tight clothing so that the movement of the limbs is restricted invariably become rickety, unless the diet is correspondingly reduced.

The avoidance of infections, catarrhal and otherwise, is an essential element in prophylactic treatment, but more easily enjoined than carried out. In healthy, well-fed infants, infections, though they inevitably occur, soon run their course, and it must be remembered that it is chronic and not short-lived acute attacks that lead to the chronic conditions of acidosis which deprive growing bones of their mineral elements.

As for remedial treatment, this depends largely on the correct diagnosis of the cause of the acidosis, on the treatment of the acidosis itself, and on the management of the complications arising from the softening of bone and of the emergencies which are secondary to the acidosis.

The more common causes of the acidosis of rickets have been already indicated, and it is unnecessary to describe in detail the specific measures called for in each particular case. The treatment of the acidosis is by no means a simple task, especially in severe cases with air-hunger, Cheyne-Stokes breathing, tetany, laryngismus stridulus, or convulsions. There are, however, certain important indications, which are as follows:

1. To restore the reduced alkali reserves of the blood.

2. To ensure a free supply of oxygen, and relieve the embarrassment of the respiratory centres.

3. To raise the blood pressure.

4. To promote the excretory functions of the kidneys.

5. To suspend temporarily the intake of food.

6. To restore muscular tone by massage and shower baths.

These, with the exception of No.6, are the measures which have: been empirically proved to be efficacious in such extreme cases of acute acidosis as accur in cholera infantum, diabetic coma, and uraemic conditions.

The reduced alkali reserves and the fall in blood pres sure can both be relieved by the intravenous or subcutaneous injeetion of isotonic saline, or, better, by a 5 per cent. solution of sodium bicarbonate and sodium citrate. Oxygen ean be supplied by inhalations, best by Haldane's continuous method (see British Medical Journal, July 19th, 1919, p. 70).

The excretory functions of the kidneys can be promoted by such cardiac stimulants as camplior, digitalis, strychnine, alcohol, caffeine, etc., or by such direct stimulants as scoparium and turpentine.

The treatment of deformities and mechanical disabilities due to softness of bone need not here be considered beyond referring to the uselessness of splints for the correcicicn of deformities of the legs, if these splints interfere with the one grand prophylactic-namely, the taking of exercise.

The treatment of emergencies, such as laryngismus stridulus, convulsions, and tetany, is as a rule satisfactory and immediate, if conducted on rational lines directed to counteract the effects of a severe acidosis.

In all cases of rickets in which the nervous symptoms are prominent I have found that phosphorated cod-liver oil ( 1 in 10,000) affords most excellent results. The phosphorus may help to restore degenerated nerve cells. I cannot, however, regard it as a general specific for all forms of rickets, as has been claimed by certain German authorities.

In conclusion, I would once again emphasize that all varieties of malnutrition, from whatsoever causes arising, in infants ultimately lead to a condition of acidosis and the characteristic bony changes. Each variety has its own etiology and requires a special line of treatment.

REFERENCE.
John Howland, Johns Hopkins Hosp. Bull., October, 1918, p. 233.

\section{RAT DESTRUCTION}

$$
\text { BY }
$$

F. NORMAN WHITE, M.D., C.I.E.,

MAJOR I.M.S., LATELX SANITARY COMMIBgIONER WITH THE GOVERNMENT IN INDIA.

IN view of the interest now being taken in England in the subject of rat destruction I have been asked to place or record some of the results of Indian experience, mor especially as regards rat poisoning. It is almost unneces sary to remind the reader that the "rat problem" has been for many year :, and is, a problem of the highest publi health importance in India; bubonic plague, so essentiall a rat disease, has been responsible for upwards of ten anc a half million deaths singe the invasion of India by plagu in 1896.

Though Indian experience may well be of assistance te those undertaking rat campaigns in England, it must not be forgotten that in India our greatest efforts have to be directed against the black rat, Rattus rattus, whereas in England the brown rat, Rattus norvegicus, is responsible for most of the damage done. The habits of the two species present striking differences. Rattus rattus, formerly the common rat of England, is now rarely seen outside our ports and large towns; its disappearance is generally ascribed to the advent of $R$. norvegicus. It would seem probable, however, that changes in the customs and habits of the people have had quite as much, if not more, to do with the elimination of $R$. rattus; both species flourish exceedingly in certain eastern cities such as Bombay, where there does not appear to be any marked antagonism between the two species. Their haunts differ it is true, but they have their common meeting places.

This short article deals chiofly with rat destruction by means of poison. It must not be concluded that this, or all the means of destruction put together, is the most important measure that can be adopted in our efforts directed to the elimination of the rat. The protection of foodstuffs from the depredations of rats; efficient scavenging; the proper construction of warehouses, stores markets, shops, granaries, dwelling houses, etc. - all such measures are likely to prove of more permanent benefit and even cheaper in the long run. A direct attack on the rat involves a constant struggle against the remarkable fecundity characteristic of all species of the genus. As a temporary measure, however, rat destruction may be an imperative necessity, notably in the event of a threatened outbreak of a rat-borne disease.

Many well-intentioned and energetieally-prosecuted rat campaigps in India and doubtless in this country also, have yielded disappointing results owing to too little 